\title{
EVALUACIÓN DE DOSIS Y MÉTODOS DE APLICACIÓN DE AZUFRE Y SU EFECTO RESIDUAL EN EL CULTIVO DE MAÍZ EN DOS LOCALIDADES DE AZUERO, PANAMÁ*
}

\author{
Román Gordón **, Andrés González **, Jorge Franco **, \\ Nivaldo De Gracia**, Adys de Herrera**, William Raun***
}

\begin{abstract}
RESUMEN
En el área de Azuero, Panamá; se observó la respuesta del maíz al efecto residual de azufre (CaS04). Los tratamientos en 1989 consistieron en dos métodos de aplicación de azufre (voleo y chuzo) y cuatro dosis de azufre de $0,20,40$ y $80 \mathrm{~kg} / \mathrm{ha}$ (cuando el método fue al voleo) y tres dosis de azufre de 0,20 y $40 \mathrm{~kg} / \mathrm{ha}$ (cuando el método fue a chuzo). En el segundo ciclo (1990) a las parcelas sólo se les aplicó 100 $\mathrm{kg}$ de Nitrógeno/ha, con el fin de obselVar el efecto residual de los tratamientos. En 1989 hubo respuesta lineal con la adición dfe yeso, tanto en forma de voleo como a chuzo, pero no se presentó una interacción entre estas dos variables independientes. Con las dosis de azufre $20 \mathrm{~kg} / \mathrm{ha}$ se obtuvo uno de los mejores rendimientos. La respuesta residual de aplicar azufre (1990) fue altamente significativa, obteniéndose incrementos en la producción que van de 0,483 hasta 1.682 t/ha. El análisis económico conjunto de los dos ciclos (1989-90) demostró que la aplicación de azufre fue rentable respecto a la dosis cero, dado los bajos costos del yeso agrícola.
\end{abstract}

\begin{abstract}
Two trials were conducted in 1990 in the zone of Azuero-Panama, to observe the response of the corn to the residual effect of sulphur (CaS04) under two application methods. In 1989, sulphur was broadcasted at arate of $0.20,40$ and $80 \mathrm{~kg} / \mathrm{ha}$, or handspiked at arate of 0.20 and $40 \mathrm{~kg} / \mathrm{ha}$. During the second cycle (1990), only $100 \mathrm{~kg} / \mathrm{ha}$ of N were applied to the plots in order to observe the residual effect of the treatments. There was a lineal response for the two bypsum application methods in the first cycle, but there was no interaction among these two independent variables. The fact that one of the. highest yields was obtained with a $20 \mathrm{~kg} / \mathrm{ha}$, doseshows us that a low dosage of this element is anough to have significant responses. The resiudal response of the sulphur application (1990) was highly significant getting yield increments ranging from 0.483 to $1.682 \mathrm{t} / \mathrm{ha}$. The economic analysis of the two cycles (1989-1990) showed that the sulphur application was profitable with respect to the dose 0 (zero), given the low cost of the agricultural gypsum.
\end{abstract}

\section{INTRODUCCION}

Las plantas necesitan por lo menos 16 nutrimentos para su desarrollo normal. El nitrógeno, el fósforo y el potasio son conocidos como los nutrimentos principales; pero otro elemento que es requerido en grandes dosis lo es el azufre. Este nutrimento es un componente esencial de algunas vitaminas, enzimas y proteínas de ahí su importancia para las plantas. En suelos que son deficientes en azufre, tanto el rendimiento como la calidad de los cultivos, se reducen si no se aplica este elemento. Niveles cr iticos en tejidos de plantas de maíz fueron determinados pero en éstos se encontró una baja correlación con estudios de campo, Kang y Osiname (1976), Daigger y Fax (1971). En Panamá, observaciones de campo han mostrado que en el cultivo de maíz se presentan deficiencias de este elemento.
Estudios realizados han mostrado que las respuestas al azufre interaccionan antagónicamente con el fósforo y el calcio, Barrow (1969), Ensminger (1954), Kamprath et al. (1956), Pasricha y Aulakh (1986). En el trópico las pérdidas de este elemento son muy variadas. pero se ha encontrado que el $90 \%$ de las bases solubles en el agua en perfiles de suelos ácidos fueron identificadas como sulfatos.

El objetivo de este trabajo fue evaluar el efecto residual de varias dosis de azufre bajo dos métodos de aplicación.

\section{MATERIALES Y MÉTODOS}

Se realizaron dos ciclos de un experimento en la Región de Azuero, República de Panamá. El primer ciclo se sembró en las localidades de Parita, Las Tablas y La

\footnotetext{
* Trabajo presentado a la XXXVII Reunión Anual del PCCMCA, Panamá. 1991.

** IDIAP. Panamá. Centro Regional de Azuero.

*** CIMMYT, Guatemala.
} 
Honda de agosto a diciembre de 1989 (época lluviosa) y un segundo ciclo en 1990, en las localidades de Parita y La Honda en la misma época. El diseño experimental utilizado fue el de bloques completos al azar con cuatro repeticiones. El tamaño de las parcelas experimentales consistió de una parcela de cuatro surcos de $5,5 \mathrm{~m}$ de largo. La población de maíz en ambos ciclos fue de aproximadamente 53,000 plantas/ha; ésto se consiguió con una distancia de siembra de $0,75 \mathrm{~m}$ entre hileras y $0,50 \mathrm{~m}$ entre golpes. En cada golpe se dejaron dos plantas.

Se utilizó la variedad X-3214, sus semillas se trataron con el insecticida furatiocarb, a razón de 10 g.i.a/ $/ \mathrm{kg}$ de semilla. En las tres áreas el suelo se clasifica como orden Alfisol sub-orden ustalf.

En el ciclo 89 se aplicaron Sulfato de Amonio (NH4)2S04 y la otra el yeso (CAS04). Se evaluaron dos métodos de aplicación uno al volea y el otro en banda. Estos dos métodos de aplicación se combinaron en un diseño factorial con tres dosis de $\mathrm{S}$ cuando se aplicó en banda $(0,20$ y $40 \mathrm{~kg} / \mathrm{ha})$ y cuatro dosis de $\mathrm{S}(0,20,40$ y $80 \mathrm{~kg} / \mathrm{ha}$ ) cuando la aplicación fue al volea. Todos los tratamientos llevaban $108 \mathrm{~kg}$ de N/ha más $52 \mathrm{~kg} / \mathrm{ha}$ de P205 juntos en una misma banda. Los tratamientos que llevaban el yeso en banda iban junto al nitrógeno y al fósforo. Además, de la aplicación de estos 12 tratamientos se añadieron nueve tratamientos no factoriales que fueron:

a) Tres tratamientos con yeso $(40 \mathrm{~kg} / \mathrm{ha})$ aplicados en banda junto al N; uno con el fósforo aplicado al voleo (T13), el otro aplicando el fósforo en una banda aparte del $\mathrm{N}$ y S (T14) y otro sin fósforo (T18).

b) Uno sin S, con una aplicación de N y P en bandas separadas (T15).

c) Dos con aplicación de yeso (40 kg/ha), uno junto al P pero en banda separada al N (T16) y el otro separado en una banda del P y N (T17).

d) Tres tratamientos con un nivel de $40 \mathrm{~kg}$ de $\mathrm{S} / \mathrm{ha}$, en donde la fuente fue el Sulfato de Amonio. Uno sin P (T19), otro junto al P en una misma banda (T20) y otro separado del P (T21).

La fuente del nitrógeno fue la urea (46-0-0). Esta se aplicó un tercio a la siembra y dos tercios 35 días después de la siembra (dds) (Cuadro 1).
Cuadro 1. Descripción de los tratamientos empleados para evaluar el efecto residual del azufre sobre el maíz. Azuero, Panamá. (1990).

\begin{tabular}{lccl}
\hline \multirow{2}{*}{$\begin{array}{c}\text { No. de } \\
\text { Tratamiento }\end{array}$} & \multicolumn{2}{c}{ CaSo4 $(\mathrm{kg} / \mathrm{ha})$} & \\
\cline { 2 - 3 } & VOLEO & BANDA & N Y P \\
\hline 1 & 0 & 0 & Banda junto \\
2 & 0 & 20 & Banda junto \\
3 & 0 & 40 & Banda junto \\
4 & 20 & 0 & Banda junto \\
5 & 20 & 20 & Banda junto \\
6 & 20 & 40 & Banda junto \\
7 & 40 & 0 & Banda junto \\
8 & 40 & 20 & Banda junto \\
9 & 40 & 40 & Banda junto \\
10 & 80 & 0 & Banda junto \\
11 & 80 & 20 & Banda junto \\
12 & 80 & 40 & Banda junto \\
13 & 0 & 40 & (N S) P Vo1 \\
14 & 0 & 40 & (N S) (P) \\
15 & 0 & 40 & (N) (P) \\
16 & 0 & 40 & (N) (P S) \\
17 & 0 & 40 & (N P) (S) \\
18 & 0 & 40 & (N S) Sin P \\
19 & 0 & 40 & (N) Sin P \\
20 & 0 & 40 & (N P) \\
21 & 0 & 40 & (N) (P) \\
\hline
\end{tabular}

40 - Sulfato de Amonio usado como fuente de Azufre. Fuente de $\mathrm{N}=$ Urea; Fuente de $\mathrm{P}=$ Super Fosfato Triple.

En las localidades de Parita y Las Tablas la preparación del suelo consistió de un pase de arado y dos pases de rastra labranza convencional y al control de malezas se realizó con la mezcla de atrazina más pendametalin $(2+2 \mathrm{l} / \mathrm{ha})$, además de limpieza manual. La Honda se utilizó el sistema de labranza de conservación que consiste en el chapeo de la maleza y la posterior aplicación de paraquat al momento de la siembra.

Para el segundo ciclo (90) las parcelas del ensayo se dejaron marcadas desde el año anterior y se procedió a sembrar el maíz sobre la línea (tratando de que los sitios de siembra coincidieran de un año a otro). La fertilización consistió de la aplicación de $100 \mathrm{~kg}$ de Nitrógeno en dos partes, 50\% al momento de la siembra y el resto a los 35 dds. En este segundo año sólo se le pudo realizar el seguimiento (residual) a los ensayos que se sembraron en Parita y La honda. El tipo de labranza para ambas localidades fue el de Conservación.

En ambos ciclos se tomaron los datos de rendimiento, número de plantas cosechadas, número de mazorcas y porcentaje de humedad del grano a los dos surcos centrales (parcela efectiva). Se tomaron los registros de precipitación pluvial de las tres áreas. Los datos obtenidos 
fueron analizados por localidad y se realizó un análisis combinado de las tres localidades. Además se realizó un análisis de varianza de cada localidad y las medias se separaron utilizando el método de contrastes no ortogonales. El análisis de suelo por localidad se observa en el Cuadro 2. Se efectuó una evaluación económica conjunta de los dos ciclos (incorporando el efecto residual) para las dos localidades, utilizando la técnica de presupuesto parcial y el análisis marginal que relaciona los beneficios netos con los costos que varían asociados a cada tratamiento (CIMMYT, 1980).

Cuadro 2. Principales características químicas de los suelos en tres localidades. Azuero, Panamá. 1990.

\begin{tabular}{lccccccc}
\hline & $\mathrm{pH}$ & $\begin{array}{c}\mathrm{p} \\
\text { LOCALIDAD }\end{array}$ & $\begin{array}{c}\mathrm{Ca} \\
\mathrm{g} / \mathrm{ml}\end{array}$ & $\mathrm{Mg}$ & $\mathrm{Al}$ & $\%$ & \\
& & & & & \\
Parita & 5,3 & 2,50 & 1577 & 253 & 6,0 & 3,00 & FARC \\
Las Tablas & 5,2 & 2,50 & 1748 & 734 & 6,0 & 2,90 & FARC \\
La Honda & 5,8 & 4,23 & 1165 & 405 & 6,0 & 2,30 & FARC \\
\hline pH $=1: 2.5$ (agua) & & & & & \\
$\mathrm{P}=$ Molich 1 & & & & & \\
Ca, Mg = KCI 1N \\
M.O. = Walkey y Black
\end{tabular}

\section{RESULTADOS Y DISCUSIÓN}

\section{Primer ciclo (1989)}

Todas las medias de los rendimientos por cada tratamiento se encuentran en el Cuadro 3. Hubo pocas diferencias entre los tratamientos en las tres diferentes localidades.

Cabe señalar que las deficiencias de azufre se observan principalmente en las hojas más jóvenes cuando las plantas estaban en las primeras etapas. Esto se debe, principalmente a que en las épocas tardías de la planta, ya existe un sistema radicular bien desarrollado, lo que le permite explorar un mayor volumen de suelo y así absorber mas azufre. Aunado a ésto, la mineralización de los rastrojos del ciclo anterior, principalmente en forma de ésteres se incrementa con la llegada de las lluvias.

El análisis combinado de las localidades demostró que hubo efectos significativos, al aplicar el sulfato de calcio (yeso) al voleo y en banda junto con el $\mathrm{N}$ y el $\mathrm{P}$. Cuando se analizaron por separados las tres localidades, este efecto no se notó con la misma significancia.

En el análisis de los tratamientos factoriales por localidad no hubo significancia de los tres modelos independientes. Se observó que hubo una respuesta lineal de las aplicaciones de yeso al voleo. También hubo efecto lineal con la aplicación de yeso al voleo, pero no se presentó una interacción entre estas dos variables.

Cuadro 3. Medias del rendimiento de maíz de los dos ciclos del cultivo en 3 localidades de Azuero, Panamá. 1989-1990.

\begin{tabular}{|c|c|c|c|c|c|c|c|c|}
\hline \multicolumn{3}{|c|}{ APLICACION DE S } & \multicolumn{6}{|c|}{ RENDIMENTO ( $t / h a)$} \\
\hline \multirow{2}{*}{ Trat } & ( kg & $\mathrm{S} / \mathrm{ha})$ & PARI & ITA & LA $\mathrm{F}$ & HONDA & LA I & HONDA \\
\hline & VOLEO & BANDA & 1989 & 1990 & 1989 & 1990 & 1989 & 1990 \\
\hline 1 & 0 & 0 & 5,14 & 6,16 & 5,03 & 5,78 & 5,09 & 5,97 \\
\hline 2 & 0 & 20 & 5,25 & 6,20 & 5,35 & 5,52 & 5,30 & 5,86 \\
\hline 3 & 0 & 40 & 5,56 & 6,90 & 5,69 & 5,91 & 5,63 & 6,41 \\
\hline 4 & 20 & 0 & 5,11 & 6,17 & 4,96 & 5,22 & 5,04 & 5,70 \\
\hline 5 & 20 & 20 & 5,68 & 6,18 & 5,77 & 6,06 & 5,73 & 6,12 \\
\hline 6 & 20 & 40 & 5,27 & 6,43 & 5,37 & 5,46 & 5,32 & 5,95 \\
\hline 7 & 40 & 0 & 4,57 & 6,57 & 5,79 & 5,60 & 5,18 & 6,09 \\
\hline 8 & 40 & 20 & 5,42 & 7,85 & 5,99 & 6,33 & 5,71 & 7,09 \\
\hline 9 & 40 & 40 & 5,11 & 5,81 & 5,36 & 5,65 & 5,24 & 5,73 \\
\hline 10 & 80 & 0 & 5,52 & 6,93 & 5,39 & 5,53 & 5,46 & 6,23 \\
\hline 11 & 80 & 20 & 6,07 & 6,30 & 5,23 & 6,50 & 5,65 & 6,40 \\
\hline 12 & 80 & 40 & 5,87 & 6,46 & 5,67 & 5,41 & 5,77 & 5,94 \\
\hline 13 & 0 & 40 & 5,41 & 6,43 & 5,22 & 6,01 & 5,32 & 6,22 \\
\hline 14 & 0 & 40 & 5,20 & 6,65 & 4,96 & 5,94 & 5,08 & 6,30 \\
\hline 15 & c & 0 & 5,41 & 6,00 & 5,11 & 6,10 & 5,26 & 6,05 \\
\hline 16 & 0 & 40 & 5,89 & 6,60 & 5,71 & 5,98 & 5,80 & 6,29 \\
\hline 17 & 0 & 40 & 5,15 & 6,18 & 5,92 & 6,02 & 5,54 & 6,10 \\
\hline 18 & 0 & 40 & 4,91 & 5,80 & 5,72 & 5,41 & 5,32 & 5,61 \\
\hline 19 & 0 & $* 40$ & 4,63 & 5,78 & 5,23 & 5,41 & 4,93 & 5,60 \\
\hline 20 & 0 & $\approx 40$ & 5,01 & 5,91 & 5,74 & 6,19 & 5,38 & 6,05 \\
\hline 21 & 0 & $* 40$ & 5,52 & 6,70 & 5,56 & 5,73 & 5,54 & 6,22 \\
\hline
\end{tabular}

A pesar de que el efecto del voleo fue significativo al $10 \%$ en el modelo, el contraste de la respuesta lineal fue significativo al $5 \%$ de probabilidad.

Con el efecto lineal de la adición al voleo, se obtuvieron rendimientos de $5,22,5,50,5,51$ y $5,54 \mathrm{t} / \mathrm{ha}$ para las dosis de $0,20,40$ y $80 \mathrm{~kg}$ de $\mathrm{S}$ como sulfato de calcio/ha. Aunque las diferencias en los rendimientos no fueron muy grandes $(300 \mathrm{~kg} / \mathrm{ha})$ el análisis combinado confirmó que estas diferencias fueron significativas. El rendimiento obtenido con la dosis de $20 \mathrm{~kg}$ de $\mathrm{S} / \mathrm{ha}$ fue uno de los mejores; lo que sugiere que solo se necesita una dosis bastante baja para obtener una respuesta por la aplicación de este elemento.

En la aplicación de $\mathrm{S}$ en banda junto con el $\mathrm{P}$ y el $\mathrm{N}$ (a través de las dosis al voleo) se obtuvieron rendimientos de 5,29, 5,50 y 5,54 t/ha para los niveles 0,20 y $40 \mathrm{~kg}$ de $\mathrm{S} / \mathrm{h}$. Como se habló anteriormente la falta de interacción voleo por banda indica que la aplicación de $20 \mathrm{~kg}$ de $\mathrm{S}$ junto con el $\mathrm{P}$ y $\mathrm{N}$ en banda ó $20 \mathrm{~kg}$ de $\mathrm{S}$ al voleo, dan resultados similares y los máximos rendimientos. 
Hubo una interacción entre localidades y la aplicación del azufre al voleo. En La Honda los rendimientos aumentaron hasta alcanzar el nivel de 40 $\mathrm{kg} / \mathrm{ha}$, para luego bajar cuando el nivel fue de $80 \mathrm{~kg}$, por el contrario en Las Tablas el máximo rendimiento se logró a un nivel de $20 \mathrm{~kg} / \mathrm{ha}$ y luego se redujo. El análisis combinado de localidades detectó diferencias entre los tratamientos, pero no se observó una interacción entre localidad por tratamiento.

El beneficio de haber aplicado el S, se observó comparando los tratamientos T3-T15 vs el T16-T17 tratamientos que no mosotraron diferencias significativas en sus contrastes.

Es importante notar que la respuesta al $\mathrm{S}$ estuvo influida por el método de aplicación. Los mejores resultados se obtuvieron con $120 \mathrm{~kg}$ de azufre como su1fato de calcio aplicado junto con el fósforo y el nitrógeno a la siembra, y con $20 \mathrm{~kg}$ de azufre aplicado al voleo. Similarmente, con $40 \mathrm{~kg}$ de azufre aplicado en banda con el fósforo aparte del nitrógeno mostró que se pueden obtener rendimientos. Por el contrario el azufre aplicado en banda junto con el $\mathrm{N}$ y el $\mathrm{P}$ aplicado al voleo (T13) no superó al testigo sin azufre. Las diferencias entre métodos de aplicación se atribuyen a que había respuesta no solo al azufre, si no que también al fósforo. Respuesta que en otros trabajos demuestran que interactuan antagónicamente, Barrow (1969), Ensminger (1954), Kamprath et al. (1956) Pasricha y Aulakh (1986).

Con relación a las fuentes de azufre se determinó que no hubo diferencias entre el sulfato de amonio y el yeso.

Se observó que hubo respuesta a la aplicación de fósforo al comparar el tratamiento que no tenían fósforo (T18 y T19) contra otros (T3, T16 y T20), esto nos demuestra que en el área este elemento es deficitario. Esta respuesta se observó con mayor énfasis cuando se comparó contra los tratamientos que llevaban juntos en banda el fósforo y el azufre.

\section{Segundo ciclo (1990)}

La respuesta residual de aplicar azufre (1990) fue altamente significativa, obteniéndose incrementos en la producción que van de 0,483 hasta 1,682 t/ha (Cuadro 3). Esto indica de que en un segundo año este elemento cobró mayor importancia en comparación con los resultados obtenidos en un primer año. Sería interesante seguir evaluando por un ciclo residual adicional (tercer ciclo) para observar la tendencia en la respuesta al azufre.

En análisis económico que relaciona los beneficios y costos que varian en cada alternativa tecnológica, se efectuó de manera conjunta incorporando los dos ciclos evaluados. Se consideró la sumatoria de los costos que var ían y los beneficios del primer ciclo más los costos y beneficios actualizados (utilizando una tasa de descuento del $15 \%$ que corresponde al costo del capital en el área) del ciclo residual. En los Cuadros 4 y 5 se presentan el detalle de los beneficios y costos correspondientes a los dos ciclos. Los costos fueron calculados con base a la estructura de tratamientos.

Cuadro 4. Descripción de los costos variables en Balboas por hectárea del ensayo de evaluación de métodos de aplicación de azufre y su efecto residual en maíz. Azuero, Panamá. 1989-199

\begin{tabular}{|c|c|c|c|c|c|c|c|c|c|c|c|}
\hline \multirow{2}{*}{$\begin{array}{c}\text { Trat } \\
\\
1 \\
2 \\
3\end{array}$} & \multirow{2}{*}{$\begin{array}{l}\text { costos } \\
\text { vol } \\
\text { (1) }\end{array}$} & \multirow{2}{*}{$\begin{array}{l}\text { costo } \\
\text { Band } \\
\text { (1) }\end{array}$} & \multirow{2}{*}{\multicolumn{3}{|c|}{ 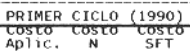 }} & & \multicolumn{4}{|c|}{ SEGUNDO CICLO (1990) } \\
\hline & & & & & & 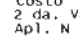 & $\begin{array}{l}\text { vartab. } \\
\text { vatal. } \\
\text { Total }\end{array}$ & $\underset{\mathrm{Ne}}{\mathrm{Ne}}$ & ${ }_{N}^{A P I C}$ & $\begin{array}{l}\text { varpab.v } \\
\text { Total }\end{array}$ & $\begin{array}{l}\text { Varlab } \\
\text { Actuai }\end{array}$ \\
\hline $\begin{array}{l}1 \\
2 \\
3 \\
4 \\
5 \\
6 \\
6 \\
7 \\
8 \\
9 \\
10 \\
11 \\
12 \\
13 \\
11 \\
15 \\
115 \\
17 \\
18 \\
19 \\
20 \\
21\end{array}$ & $\begin{array}{c}0 \\
0 \\
0 \\
0 \\
55,40 \\
5,40 \\
5,40 \\
10,80 \\
10,80 \\
10 ;, 80 \\
21,60 \\
21,60 \\
21,60 \\
0 \\
0 \\
0 \\
0 \\
0 \\
0 \\
0 \\
0 \\
0 \\
0\end{array}$ & $\begin{array}{c}0 \\
5,40 \\
10,80 \\
5,40 \\
5,40 \\
10,80 \\
5,40 \\
10,80 \\
5,40 \\
10,80 \\
10,80 \\
10,80 \\
10,80 \\
10,80 \\
10,80 \\
10,80 \\
38,40 \\
38,40 \\
38,40\end{array}$ & $\begin{array}{c}0 \\
0 \\
0 \\
0 \\
2,50 \\
2,50 \\
2,50 \\
2,50 \\
5,00 \\
5,00 \\
5,00 \\
10 ; 00 \\
10 ; 00 \\
10 ; 00 \\
2,50 \\
2,50 \\
2,50 \\
7,00 \\
5,00 \\
0 \\
0 \\
0 \\
0 \\
2,50\end{array}$ & $\begin{array}{l}66,00 \\
66,00 \\
66,00 \\
66,00 \\
66,00 \\
66,00 \\
66,00 \\
66,00 \\
66,00 \\
66,00 \\
66,00 \\
66,00 \\
66,00 \\
66,00 \\
66 ; 00 \\
66,00 \\
66,00 \\
66,00 \\
44,70 \\
44,70 \\
44,70\end{array}$ & $\begin{array}{r}41,08 \\
41,08 \\
41,08 \\
41,08 \\
41,08 \\
41,08 \\
41,08 \\
41,08 \\
41,08 \\
41,08 \\
411,08 \\
41,08 \\
411,08 \\
411,08 \\
411,08 \\
441,08 \\
41,08 \\
0 \\
0 \\
41,08 \\
41,08 \\
41,08 \\
\end{array}$ & $\begin{array}{l}3,50 \\
3,50 \\
3,50 \\
3,50 \\
3,50 \\
3,50 \\
3,50 \\
3,50 \\
3,50 \\
3,50 \\
3,50 \\
3,50 \\
3,50 \\
3,50 \\
3,50 \\
3,50 \\
3,50 \\
3,50 \\
3,50 \\
3,50 \\
3,50 \\
3,50\end{array}$ & 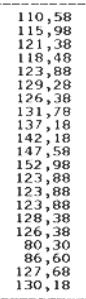 & & 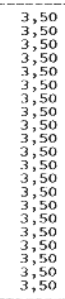 & $\begin{array}{l}49,50 \\
49,50 \\
49,50 \\
49,50 \\
49,50 \\
49,50 \\
49,50 \\
49,50 \\
49,50 \\
49,50 \\
49,50 \\
49,50 \\
49,50 \\
49,50 \\
49,50 \\
49,50 \\
49,50 \\
49,50 \\
49,50 \\
49,50 \\
49,50\end{array}$ & 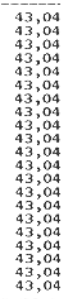 \\
\hline
\end{tabular}

Cuadro 5. Beneficios y costos en Balboas por hectárea del ensayo de evaluación de dosis y métodos de aplicación de azufre y su efecto residual en maíz. Azuero, Panamá. 1989-1990.

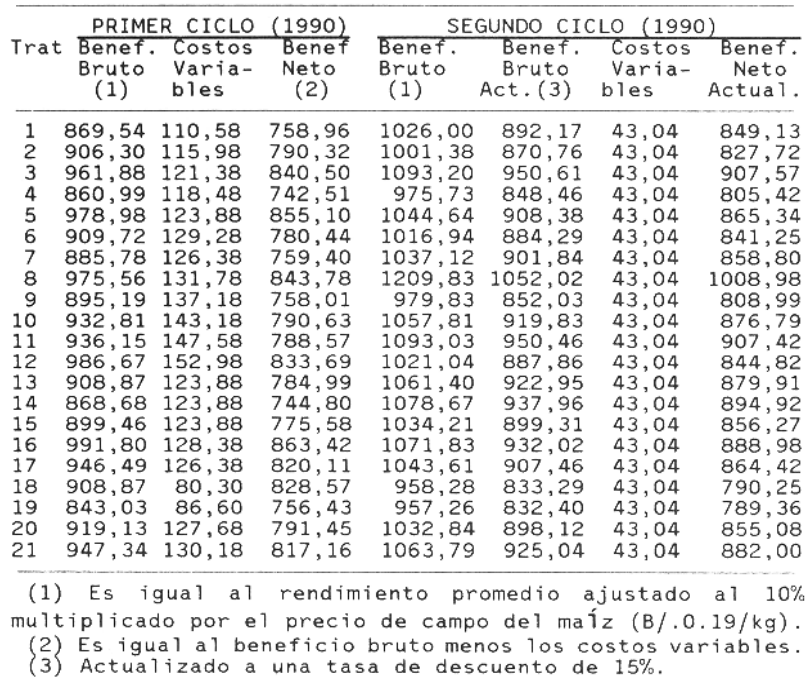


El Cuadro 6, describe el análisis económico marginal, el cual fue realizado solamente con los tratamientos factoriales. La aplicación del azufre fue altamente rentable, medido a través de los mayores beneficios netos obtenidos respeto al nivel cero (T1) y que constituye la práctica de los agricultores del área. Esto lo explica el incremento significativo en los rendimientos obtenidos con la aplicación del azufre, y el bajo precio internacional del yeso agricola. El yeso agrícola no está disponible en el mercado panameño, por lo que se utilizó el precio de este producto en Guatemala. No obstante, las altas tasas marginales de retorno obtenidas implican que aún, si el precio fuera considerablemente más alto (asumiendo que se importara el mismo) se justificar ia económicamente su utilización.

Cuadro 6. Análisis económico marginal conjunto (dos ciclos) del ensayo de evaluación de dosis y métodos de aplicación de azufre y su efecto residual en maíz. Azuero, Panamá. 1989-1990.

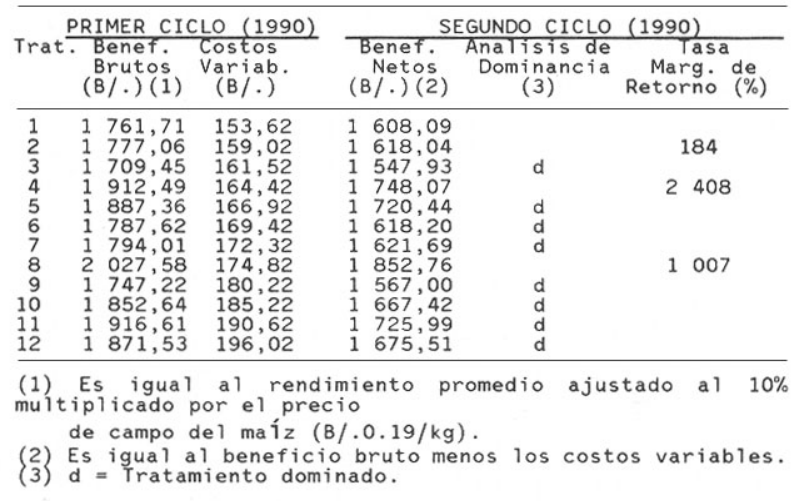

El efecto residual de aplicar el yeso junto con el fósforo juntos en una misma banda, tuvo un incremento de $300 \mathrm{~kg} / \mathrm{ha}$, sobre la parcela que tenía estos dos elementos en bandas separadas, pero la misma no fue significativa. Se observó que hubo respuesta altamente significativa a la aplicación de fósforo, lo que reafirma los resultados obtenidos en el primer ciclo.

\section{CONCLUSIONES Y RECOMENDACIONES}

En los dos años hubo una respuesta altamente significativa a la aplicación de azufre, obteniéndose incrementos de 0,33 a $0,88 \mathrm{t} /$ ha el primer ciclo y de 0,483 hasta $1,682 \mathrm{t} / \mathrm{ha}$.

El análisis económico conjunto de los dos años demostró alta rentabilidad en la aplicación de azufre versus la no aplicación.
La respuesta de aplicar azufre al voleo y en banda fue lineal en 1989 y cuadrática al voleo en el ciclo residual (1990).

En el segundo ciclo de cultivo se observó una interacción entre el método de voleo por banda; siendo el tratamiento de $40 \mathrm{~kg}$ S/ha al voleo por $20 \mathrm{~kg}$ S/ha en banda el de mayor Beneficio Neto por hectárea y una Tasa Marginal de Retorno (100\%), ampliamente superior a la Tasa Minima de Retorno de los productores de la Región.

La dosis de $20 \mathrm{~kg}$ de azufre/ha produjo uno de los rendimientos más altos, lo que nos indica que una dosis baja es suficiente para obtener rendimientos óptimos en el primer ciclo.

No hubo diferencias entre las fuentes de azufre (Sulfato de Calcio y Sulfato de Amonio).

Se observó una respuesta significativa de aplicar fósforo en los dos ciclos de cultivo de este experimento.

No se observó ninguna ventaja de aplicar el yeso junto al azufre en los dos ciclos del cultivo; solo se obtuvo un incremento de $300 \mathrm{~kg} / \mathrm{ha}$ en el ciclo residual, pero el mismo no fue significativo.

\section{LITERATURA CITADA}

BARROW, N. J. 1969. Effects of adsorption of sulfate by soils on the amount of sulfate present and ist availability to plants. Soil Sci. 108:193-201.

CENTRO INIERNACIONAL DE MEJORAMIENrO DE MAIZ Y TRIGO. 1988. La formulación de recomendaciones a partir de datos agronómicos: Un manual metodológico de evaluación económica. Edición completamente revisada. México D.F. méxico: CIMMYT 79p.

DAIGGER, L.A.; FOX, R. L 1971. Nitrogen and sulfur nutrition of sweet corn in relation to fertilization and water composition. Agron. J. 63:729-730.

ENSMINGER, L E. 1954. Some factors affecting the adsorption of sulfate by Alabama soils. Soil. Soc. Am. Proc. 18:259-264.

KAMPRATH, E. J.; W. L NELSON; FITTS, J. W. 1956. The effect of $\mathrm{pH}$, sulfate and phosphate concentration on the adsorption of sulfate by soils. Soil. Sci. Soco Am. Proc. 20:463-466.

KANG, B.T.; OSINAMA, O.A 1976. Sulfur response of maize ir Western Nigeria. Agron. Journ. 68: 333-336.

PASRICHA, N.S.; AULAKH, M.S. 1986. Role of sulfur in the nutrition of groundnut. Fertilizer News. New Delhi, India. vol. 31 , no. 9 . 